PROCEEDINGS OF THE

AMERICAN MATHEMATICAL SOCIETY

Volume 140, Number 4, April 2012, Pages 1387-1392

S 0002-9939(2011)10997-3

Article electronically published on August 5, 2011

\title{
EXTREME GROWTH RATES OF PERIODIC ORBITS IN FLOWS
}

\author{
WENXIANG SUN AND CHENG ZHANG
}

(Communicated by Yingfei Yi)

\begin{abstract}
While an extreme growth rate of periodic orbits is an invariant for equivalent flows without fixed points, there exists a pair of equivalent flows with fixed points such that the growth rate of periodic orbits of one flow is infinite and that of the other is zero.
\end{abstract}

\section{INTRODUCTION}

Two flows on compact spaces are equivalent if there exists a homeomorphism of the spaces that sends each orbit of one flow onto an orbit of the other flow while preserving the time orientation. The growth rate of periodic orbits, which coincides with topological entropy in Anosov diffeomorphisms or Anosov flows [1], is a measure of complexity of a system. A pair of equivalent homeomorphisms share the periods for corresponding periodic orbits and thus share the growth rate of periodic orbits. A pair of equivalent flows might not share the same growth rates of periodic orbits, due to the time reparameterization between the two flows. We will construct a pair of equivalent flows to show the extreme situation: the growth rate of periodic orbits of one flow is infinite while that of the other flow is zero. In [5] the authors have already shown, by constructing examples, that the flow with 0 topological entropy and the flow with $\infty$ topological entropy exist simultaneously in certain classes of equivalent flows with fixed points. Nevertheless, the conclusion for the growth rate of periodic orbits is not a corollary of that for entropy in [5]. In fact, topological entropy and the growth rate of periodic orbits are different measures for complexity of a flow(although they coincide in some systems like Anosov flows). Moreover, we will construct examples to show the extreme difference: a flow may have extremely large (small) entropy and extremely small (large) growth rate of periodic orbits simultaneously.

Let $M$ be a compact metric space. Let $\phi: M \times \mathbb{R} \rightarrow M$ be a flow, a continuous map satisfying $\phi(x, 0)=x$ and $\phi(\phi(x, s), t)=\phi(x, s+t), \forall x \in M \forall s, t \in \mathbb{R}$. For given $t \in \mathbb{R}$ we denote by $\phi(\cdot, t): M \rightarrow M$ the homeomorphism $\phi(x, t)=\phi(x, t)$. Given $A \in \mathbb{R}^{+}$, we define the number of periodic orbits of at most $A$-period by

$$
\pi(\phi, A)=\max \{1, \sharp\{\operatorname{orb}(x) \subset M \text { isolated } \mid \phi(x, u)=x \text {, for some } 0 \leq u \leq A\}\} .
$$

Received by the editors August 18, 2010 and, in revised form, January 5, 2011.

2010 Mathematics Subject Classification. Primary 37C15, 34C28, 37A10.

Key words and phrases. Growth rate of periodic orbits, entropy, flow.

The first author was supported by NSFC (\#10831003) and the National Basic Research Program of China (973 Program) (\#2006CB805903) and the National Education Ministry of China. 
Let $p(\phi, A)=\frac{1}{A} \log \pi(\phi, A)$ and let $p(\phi)=\limsup _{A \rightarrow \infty} p(\phi, A)$. We call $p(\phi)$ the growth rate of periodic orbits for $\phi$. Now we state our main theorems.

Theorem 1.1. There exists a pair of equivalent flows $\phi: M \times \mathbb{R} \rightarrow M$ and $\psi$ : $W \times \mathbb{R} \rightarrow W$ with fixed points on compact metric spaces $M$ and $W$, respectively, such that

1. $p(\phi)=\infty$;

2. $p(\psi)=0$.

The huge difference happens only in the flows with fixed points. Actually, extreme growth rates of periodic orbits are invariant in equivalent flows without fixed points.

Theorem 1.2. For a pair of equivalent flows without fixed points, $\phi: M \times \mathbb{R} \rightarrow M$ and $\psi: W \times \mathbb{R} \rightarrow W$ on compact metric spaces $M$ and $W$, respectively, the following two items hold:

1. $p(\phi)=\infty \Longleftrightarrow p(\psi)=\infty$;

2. $p(\phi)=0 \Longleftrightarrow p(\psi)=0$.

In the next two propositions, we will show the extreme difference between topological entropy and the growth rate of periodic orbits.

Proposition 1.3. There is a flow $\psi$ on a compact space $W$ such that

1. $h(\psi)=\infty$;

2. $p(\psi)=0$.

Proposition 1.4. There is a flow $\phi$ on a compact space $M$ such that

1. $h(\phi)=0$;

2. $p(\phi)=\infty$.

\section{EquiVAlENT FLOWS Without FiXed POINTS}

We will prove Theorem 1.2 in this section. Before that we recall a lemma from 3.

Lemma 2.1. For a pair of equivalent flows $\phi$ and $\psi$ on compact spaces $M$ and $W$, respectively, let $\alpha: M \rightarrow W$ be a homeomorphism that sends each orbit of $\phi$ onto an orbit of $\psi$ preserving the time orientation. If there are no fixed points of $\phi$ or $\psi$, then there is a continuous function $\theta: W \times \mathbb{R} \rightarrow \mathbb{R}$ such that for $x \in M$ and $s, t \in \mathbb{R}$, the following hold:

1. $\theta(x, 0)=0$ and $\theta(x, \cdot): \mathbb{R} \rightarrow \mathbb{R}$ is strictly increasing;

2. $\theta(x, s+t)=\theta(x, s)+\theta(\phi(x, s), t)$;

3. $\alpha(\phi(x, t))=\psi(\alpha(x), \theta(x, t))$.

Proof of Theorem 1.2, Let $\alpha: M \rightarrow W$ be a homeomorphism that sends each orbit of $\phi$ onto an orbit of $\psi$ preserving the time orientation. Take a continuous function $\theta$ as in Lemma 2.1. Since $M \times[0,2]$ is compact, there is $D \in \mathbb{R}^{+}$such that $0 \leq \theta(x, t) \leq D, \forall x \in M, t \in[0,2]$. For given $t \geq 2$ we express $t=2 k+r$ for some $k \in \mathbb{N}$ and $r \in[0,2)$. According to Lemma 2.1.

$$
\begin{aligned}
\theta(x, t) & =\underbrace{\theta(x, 2)+\theta(\phi(x, 2), 2)+\cdots+\theta(\phi(x, 2 k-2), 2)}_{k}+\theta(\phi(x, 2 k), r) \\
& \leq \underbrace{D+\cdots+D}_{k}+D \leq D t .
\end{aligned}
$$


Therefore

$$
\theta(x, t) \leq D, \forall x \in M, 0 \leq t \leq 2, \quad \text { and } \quad \frac{\theta(x, t)}{t} \leq D, \forall x \in M, t \geq 2 .
$$

For $\alpha^{-1}: W \rightarrow M$, which sends each orbit of $\psi$ onto an orbit of $\phi$ preserving the time orientation, we take a time reparameterization function $\sigma(y, t)$ playing the same role of $\theta(x, t)$ as in Lemma 2.1. Similarly, there exists a constant $E>0$ such that

$$
\sigma(y, t) \leq E, \forall y \in W, 0 \leq t \leq 2, \quad \text { and } \quad \frac{\sigma(y, t)}{t} \leq E, \forall y \in W, t \geq 2 .
$$

Case 1. $\pi(\phi, 2)=\infty$.

By (2.1) it holds that $\pi(\psi, D)=\infty$. So $p(\phi)=p(\psi)=\infty$.

Case 2. $\pi(\psi, 2)=\infty$.

It holds by (2.2) that $\pi(\phi, E)=\infty$. So $p(\phi)=p(\psi)=\infty$.

In either case 1 or case $2, p(\phi)=p(\psi)$, and Theorem 1.2 holds clearly. Now we consider the other case.

Case 3. $\pi(\phi, 2)<\infty$ and $\pi(\psi, 2)<\infty$.

For given $A>2, \pi(\phi, A) \leq \pi(\psi, A D)+\pi(\psi, D)$ by (2.1).

If $p(\phi)=\infty$, then

$\infty=\limsup _{A \rightarrow \infty} p(\phi, A) \leq \limsup _{A \rightarrow \infty} D \frac{1}{D A} \log (\pi(\psi, D A)) \cdot \frac{\log (\pi(\psi, D A)+\pi(\psi, D))}{\log (\pi(\psi, D A))}=D p(\psi)$.

So $p(\phi)=\infty$ implies $p(\psi)=\infty$. Similarly by (2.2), $p(\psi)=\infty$ implies $p(\phi)=\infty$. Item 1 in Theorem 1.2 follows.

If $p(\psi)=0$, then by (2.1),

$p(\phi)=\limsup _{A \rightarrow \infty} \frac{1}{A} \log \pi(\phi, A) \leq \limsup _{A \rightarrow \infty} D \frac{1}{D A} \log (\pi(\psi, D A)+\pi(\psi, D))=D p(\psi)=0$.

So, $p(\psi)=0$ implies $p(\phi)=0$. Similarly, by (2.2), $p(\phi)=0$ implies $p(\psi)=0$. Item 2 in Theorem 1.2 follows.

Remark 2.2. We have already obtained a similar result for entropy in [4]: extreme topological entropies are invariant in equivalent flows without fixed points.

\section{EQUiVALENT FLOWS WITH FIXED POINTS}

We will construct two equivalent pointwise period flows (each point is periodic) with fixed points, of which one has zero growth rate of periodic orbits and the other has infinite growth rate of periodic orbits.

3.1. Construction of the flows. We divide the construction into two steps.

Step 1. Discrete systems.

We set $\mathbb{S}^{1}=[0,1] \bmod \mathbb{Z}$ and take a series of subsets $X_{1}=\left\{0, \frac{1}{2}\right\}, X_{2}=$ $\left\{0, \frac{1}{32}, \frac{2}{32}, \frac{3}{32}, \cdots, \frac{29}{32}, \frac{30}{32}, \frac{31}{32}\right\}, \cdots, X_{n}=\left\{0, \frac{1}{n \cdot 2^{n^{2}}}, \frac{2}{n \cdot 2^{n^{2}}}, \cdots, \frac{n \cdot 2^{n^{2}}-1}{n \cdot 2^{n^{2}}}\right\} \subset \mathbb{S}^{1}, \cdots$, where $X_{n}$ inherits the metric topology of $\mathbb{S}^{1}:\|x-y\|=\min \{|x-y|, 1-|x-y|\}$, $\forall x, y \in \mathbb{S}^{1}$. On $X_{n}$ we define a rational rotation $\rho_{n}: X_{n} \rightarrow X_{n}, \rho_{n}(x)=$ $x+\frac{1}{n} \bmod \mathbb{Z}$. Then $\left(X_{n}, \rho_{n}\right)$ is a dynamical system consisting of $n \cdot 2^{n^{2}} n$-periodic points, or in other words, $2^{n^{2}} n$-periodic orbits. 
We denote by $X$ the disjoint union of $X_{n}$ together with a point $x_{\infty}$ and we define a metric on $X$ as follows:

$d_{X}(x, y)=\frac{1}{n^{2}}\|x-y\|$ for $x, y \in X_{n}$;

$d_{X}(x, y)=\sum_{i=n}^{p} \frac{1}{i^{2}}$ for $x \in X_{n}, y \in X_{p}, n<p$;

$d_{X}\left(x_{\infty}, x\right)=\sum_{i=n}^{\infty} \frac{1}{i^{2}}$ for $x \in X_{n}$.

We claim that $\left(X, d_{X}\right)$ is a compact metric space. Suppose there is an open cover $\mathcal{U}$ of $X$ in which an element $U$ covers $x_{\infty}$; what is more, $U$ also contains a small ball $B\left(x_{\infty}, b\right)$ for some $b>0$. There exists $n \in \mathbb{N}$ such that $\sum_{i=n}^{\infty} \frac{1}{i^{2}}<b$; then $\bigcup_{i=n}^{\infty} X_{i} \subset B\left(x_{\infty}, b\right) \subset U$. Since each $X_{1} \cdots X_{n-1}$ is compact, so is their disjoint union. A subset $\mathcal{U}_{0} \subset \mathcal{U}$ with only finite elements can cover $\bigcup_{i=1}^{n-1} X_{i}$. Therefore $\mathcal{U}_{0} \cup\{U\}$ can cover $X$. Here $X$ is compact under this metric.

We define $T: X \rightarrow X$ by $\left.T\right|_{X_{n}}=\rho_{n}$ and $T\left(x_{\infty}\right)=x_{\infty}$. Clearly, $T$ is a homeomorphism on $X$.

We indicate "minimal period" by "period" from now on. In $(X, T)$, there are $2+1$ fixed points, 162 -periodic orbits, 5123 -periodic orbits,..., $2^{n^{2}} n$-periodic orbits, and all the $n$-periodic orbits are in $X_{n}, n \geq 2$.

Step 2. Suspensions.

For $r_{i}=i^{2}: X_{i} \rightarrow \mathbb{R}^{+}$, we define the quotient space $X_{i}^{r_{i}}$ of $\{(x, u): 0 \leq u<$ $\left.r_{i}(x), x \in X_{i}\right\}$ by the equivalent relation $\left(x, r_{i}(x)\right) \sim(T(x), 0)$, on which we define a flow $\psi^{i}$ by $\psi^{i}(x, u, t)=(x, u+t)$, for $-u \leq t<r_{i}(x)-u$. Then $X_{i}^{r_{i}}$ is a compact metric space and $\psi^{i}$ is a flow, called suspension flow. By one point compactification of the disjoint union of all the $X_{i}^{r_{i}}$ 's we get a compact space $W$. Without loss of generality, we still denote the compactification point by $x_{\infty}$. We define a flow $\psi$ on $W$ as $\left.\psi\right|_{X_{i}^{r_{i}}}=\psi^{i}$ for all $i$ and $\psi\left(x_{\infty}, t\right)=x_{\infty}$ for all $t \in \mathbb{R}$.

When we take $r_{i} \equiv 1$ on $X_{i}$ for each $i$, we get as above a compact metric space $M$ and a corresponding flow $\phi$. We see easily that $(M, \phi)$ and $(W, \psi)$ are topologically equivalent.

Proof of Theorem 1.1. For given $A>1$, take $n \in \mathbb{N}$ such that $n \leq A<n+1$. The flow $(M, \phi)$ has exactly 1 fixed point, 21 -periodic orbits, 162 -periodic orbits, ..., and $2^{n^{2}} n$-periodic orbits and has no extra periodic orbits with period less than or equal to $A$. So

$p(\phi, A)=\frac{1}{A} \log \pi(\phi, A)=\frac{1}{A} \log \left(1+2+16+\cdots+2^{n^{2}}\right)>\frac{1}{A} \log 2^{n^{2}}>\frac{1}{n+1} n^{2} \log 2$,

which tends to $\infty$ as $A \rightarrow \infty$, i.e. $p(\phi)=\infty$.

Now for given $B>1$, take $m \in \mathbb{N}$ such that $m^{3} \leq B<(m+1)^{3}$. The flow $(W, \psi)$ has exactly 1 fixed point, 21 -periodic orbits, 168 -periodic orbits,..., $2^{m^{2}}$ $m^{3}$-periodic orbits, and no extra periodic orbits with period less than or equal to B. So, $\pi(\psi, B)=1+2+2^{2^{2}}+2^{3^{2}}+\cdots+2^{m^{2}}<\sum_{i=0}^{m^{2}} 2^{i}=2 \cdot 2^{m^{2}}-1<2^{m^{2}+1}$. Therefore, $p(\psi, B)<\frac{1}{m^{3}} \log \left(2^{m^{2}+1}\right)$ and $p(\psi) \leq \limsup _{m \rightarrow \infty} \frac{1}{m^{3}} \log \left(2^{m^{2}+1}\right)=0$.

\section{Entropy Versus growth Rate}

We prove Proposition 1.3 and Proposition 1.4 in this section.

Proof of Proposition 1.3. We set $\Sigma_{2}=\left\{\left(\cdots s_{-1} \bar{s}_{0} s_{1} \cdots\right) \mid s_{i}=0,1, \forall i \in \mathbb{Z}\right\}$. A point $\left(\cdots s_{-1} \bar{s}_{0} s_{1} \cdots\right)$ in $\Sigma_{2}$ indicates a 2 -sided infinite sequence in which each 
position is assigned a word 0 or 1 and the 0th position is marked with a bar. We use metric topology in this space: $\|x-y\|=\sum_{n=-\infty}^{\infty}\left|x_{n}-y_{n}\right| 2^{-|n|}, \forall x, y \in \Sigma_{2}$. Clearly, $\left(\Sigma_{2},\|\cdot\|\right)$ is a compact metric space. Set $\sigma: \Sigma_{2} \rightarrow \Sigma_{2}, \sigma\left(\cdots s_{-1} \bar{s}_{0} s_{1} \cdots\right)=$ $\left(\cdots s_{0} \bar{s}_{1} s_{2} \cdots\right)$, the usual shift map.

Take $x_{*} \in \Sigma_{2}$ such that

1. for all $k \in \mathbb{Z}$ and $n \geq 1$, there are $2 n-1$ consecutive 1 's in $x_{*}(k) x_{*}(k+$ 1) $\cdots x_{*}\left(k+4 \cdot 3^{n}\right)$;

2. for all $n \geq 1$ there exist $2^{p_{n}}$ different words (sequences) of length $2 \cdot 3^{n-1}$ in $x_{*}$, where $p_{n}=\frac{3^{n-1}+1}{2}$.

The existence of such a point $x_{*}$ is guaranteed by Ohno's example in [2. For the convenience of the reader we briefly recall it as follows.

We define $x^{+} \in\{1, a\}^{\mathbb{N}}$ as follows, where $a$ is a symbol which will be replaced by 0 or 1 later. We define $x_{n}$ inductively. Put $x_{1}=1 a$ and then $x_{2}=x_{1} \tilde{x}_{1} x_{1}$ where $\tilde{x}_{1}=11$. We define $\tilde{x}_{n}$ by changing one $a$ in $x_{n}$ to 1 so that there are $2 n-1$ consecutive 1's in $\tilde{x}_{n}$ and define $x_{n+1}=x_{n} \tilde{x}_{n} x_{n}$. Let $x^{+}=\lim _{n \rightarrow \infty} x_{n}$. Then there are infinitely many $x_{n}$ in $x^{+}$for all $n \geq 1$. The number of $a$ in $x_{n}$ is $p_{n}=\frac{3^{n-1}+1}{2}$.

Changing $a$ 's in $x_{n}$ to 0 or 1 , we can obtain $2^{p_{n}}$ different words of length $2 \cdot 3^{n-1}$. We replace $2^{p_{n}} x_{n}$ 's of those words in turn from the top of $x^{+}, n=1,2 \cdots$, and put 0 or 1 arbitrarily into the remaining $a$. Thus we obtain $x^{+}$in $\{0,1\}^{\mathbb{N}}$. We put $x_{*}(k)=x^{+}(k)$ for $k \geq 1, x_{*}(0)=1$ and $x_{*}(k)=x^{+}(-k)$ for $k \leq-1$. Then it is easy to see that $x_{*}$ satisfies items 1 and 2 above.

Now the subsystem $\left(\overline{\operatorname{orb}\left(x_{*}, \sigma\right)}, \sigma\right)$ of $\left(\Sigma_{2}, \sigma\right)$ has exactly one fixed point $\tilde{1}=$ $(\cdots 1 \overline{1} 1 \cdots)$, and it has topological entropy $h(\sigma)=c \geq \lim _{n \rightarrow \infty} \frac{\log 2^{p_{n}}}{2 \cdot 3^{n-1}}=\frac{\log 2}{4}>0$.

Now we take a series of subspaces of $\Sigma_{2}: Y_{1}=\overline{\operatorname{orb}\left(x_{*}, \sigma\right)}, Y_{2}=\overline{\operatorname{orb}\left(x_{*}, \sigma^{4}\right)}, \cdots$, $Y_{i}=\overline{\operatorname{orb}\left(x_{*}, \sigma^{i^{2}}\right)}, i \in \mathbb{N}$. We denote by $Y$ the disjoint union of $Y_{i}$ together with a point $y_{\infty}$ and we define a metric on $Y$ as follows:

$d_{Y}(x, y)=\frac{1}{i^{2}}\|x-y\|$ for $x, y \in Y_{i}$

$d_{Y}(x, y)=\sum_{j=i}^{p} \frac{3}{j^{2}}$ for $x \in Y_{i}, y \in Y_{p}, i<p$;

$d_{Y}\left(y_{\infty}, y\right)=\sum_{j=i}^{\infty} \frac{3}{j^{2}}$ for $y \in Y_{i}$.

It is easy to see that $Y$ is a compact metric space. We define $T: Y \rightarrow Y$ by $\left.T\right|_{Y_{i}}:=\sigma^{i^{2}}$ and $T\left(y_{\infty}\right)=y_{\infty}$. Clearly, $T$ is a homeomorphism on $Y$. Since $h\left(\left.T\right|_{Y_{i}}\right)=i^{2} c, h(T)=\infty$.

For each $i$ we define the quotient space $Y^{i}$ of $\{(x, u): 0 \leq u \leq i\}$ by the equivalent relation $(x, i) \sim(T(x), 0)$. We also define a flow $\psi^{i}$ by $\psi^{i}(x, u, t)=(x, u+t)$, for $-u \leq t<i-u$.

Now by one point compactification of the disjoint union of all the $Y^{i}$ 's we get a compact space $W$. Without loss of generality, we still denote the compactification point by $y_{\infty}$. We define a flow $\psi$ on $W$ as $\left.\psi\right|_{Y^{i}}=\psi^{i}$ for all $i \in \mathbb{N}$ and $\psi\left(y_{\infty}, t\right)=y_{\infty}$ for all $t \in \mathbb{R}$.

Since the time- $i$ map $\psi^{i}(\cdot, i)$ of $\psi^{i}$ is topologically equivalent to $\left.T\right|_{Y_{i}}, h\left(\psi^{i}(\cdot, i)\right)=$ $h\left(\left.T\right|_{Y_{i}}\right)=i^{2} c$. So, $h\left(\psi^{i}\right)=h\left(\psi^{i}(\cdot, 1)\right)=\frac{1}{i} h\left(\psi^{i}(\cdot, i)\right)=i c$. As a result, $h(\psi)=\infty$.

There is exactly one fixed point $y_{\infty}$, one 1 -periodic orbit $\operatorname{orb}\left((\tilde{1}, 0), \psi^{1}\right)$, one 2 periodic orbit $\operatorname{orb}\left((\tilde{1}, 0), \psi^{2}\right), \ldots$, and one $i$-periodic orbit $\operatorname{orb}\left((\tilde{1}, 0), \psi^{i}\right)$ in $W, i \in \mathbb{N}$. So $p(\psi, i)=\frac{1}{i} \log (1+\underbrace{1+\cdots+1}_{i})=\frac{1}{i} \log (i+1) \rightarrow 0$, as $i \rightarrow \infty$. i.e. $p(\psi)=0$. We finish the proof of Proposition 1.3 
Proof of Proposition 1.4. Our flow $(M, \phi)$ in Subsection 3.1 is an example meeting Proposition 1.4. Indeed, that every orbit in $(M, \phi)$ is periodic implies $h(\phi)=0$ by variational principle. However, we showed in the proof of Theorem 1.2 that $p(\phi)=\infty$.

\section{REMARK}

Fixed points do play an important role in the complexity of flows. The extreme values are invariant for both topological entropy and the growth rate of periodic orbits in equivalent flows without fixed points. But neither is invariant in equivalent flows with fixed points. The examples in the present paper and [5] show that the deceleration of the flows near the fixed point causes the degeneration of entropy and the growth rate of periodic orbits. There seems to be something essential in common. However, the fact that a flow can have infinite entropy (growth rate of periodic orbits) and zero growth rate of periodic orbits (entropy) shows that there is something essentially different between them. It is interesting to measure the complexity of a system in various ways.

\section{REFERENCES}

1. R. Bowen, Periodic orbits for hyperbolic flows, Amer. Jour. Math. 94 (1972), 1-30. MR0298700 (45:7749)

2. T. Ohno, A weak equivalence and topological entropy, Publ. RIMS, Kyoto Univ. 16 (1980), 289-298. MR574037 (81g:54053)

3. V. A. Rohlin, Entropy of metric automorphism, Dokl. Acad. Nauk. USSR 124 (1959), 980-983 (in Russian). MR0103258 (21:2037)

4. W. Sun, E. Vargas, Entropy of flows, revisited, Bol. Soc. Bra. Mat. 30 (1999), 313-333. MR.1726916 (2001d:37007)

5. W. Sun and C. Zhang, Zero topological entropy versus infinite topological entropy, Dis. Con. Dyn. Sys. 30 (2011), 1237-1242.

6. P. Walters, An introduction to ergodic theory, Springer Verlag, 1982. MR648108 (84e:28017)

Lmam, School of Mathematical Sciences, Peking University, Beijing 100871, PeoPLE'S REPUBlic of ChinA

E-mail address: sunwx@math.pku.edu.cn

School of Mathematical Sciences, Peking University, Beijing 100871, People's Republic of China

E-mail address: cesariozhang@gmail.com 